\title{
Study of bacteria causing skin infections in school going children in Udupi district;cross-sectional study
}

\author{
Shobha K.L ${ }^{1 *}$, Jessica D'souza ${ }^{1}$, Varun C.N ${ }^{2}$, Vinod Kumar $^{2}$, Ramachandra Kamath ${ }^{3}$, Ramachandra L ${ }^{4}$ \\ ${ }^{l}$ Department of Microbiology,Melaka Manipal Medical College, (ManipalCampus), Manipal University, Manipal \\ ${ }^{2}$ Department of Microbiology, Kasturba Medical College, (ManipalCampus), Manipal University, Manipal \\ ${ }^{3}$ Department of Public Health,Manipal University,Manipal \\ ${ }^{4}$ Department of Surgery, Kasturba Medical College, (ManipalCampus), Manipal University, Manipal \\ *Corresponding author E-mail:shobha.kl@manipal.edu
}

\begin{abstract}
Introduction: Skin infections are a common reason for consultation in primary care and in dermatology practice. The predominant strain causing infection varies by geographical location. The aim of our study was to evaluate various types of bacteria causing skin infections in school going children of Udupi district, Karnataka State, India.

Materials and Methods: The study group consisted of school going 236 children in Udupi district. Consent was taken from the parents of respective children to screen for physical examination and to collect skin swabs. Skin swabs were collected from the site of lesions and processed for isolation and identification of the organisms. Antibacterial susceptibility testing was done by Kirby-Bauer disc diffusion test.

Results: Swabs were collected from 53 children of $236(22.45 \%)$ who had abrasions or lesions on skin. Bacterial strains isolated were Staphylococcus aureus (13.2\%) Klebsiella species (1.88\%). All the strains of Staphylococcus aureus were susceptible to Methicillin. Erythromycin resistance among these Staphylococcus aureus was $42.85 \%$

Discussion: Staphylococcus aureus, the pathogen isolated was 7 (87.5\%), this study was in concordance with the study conducted by (Cassandra D. Salgado ET al.2003), who had reported Methicillin resistant strains of 1.3\%.Our study did not find any Methicillin resistant Staphylococcus aureus. There was an increased resistance to the erythromycin. This was consistent with the data that had been established in many countries where erythromycin resistance was increasing in community acquired infections.

Conclusion: Though Staphylococcus aureus was the common organism isolated from skin swabs, all were sensitive to methicillin,but high prevalence of erythromycin resistance of the Staphylococcus aureus in the community demands proper screening of the isolates for resistance in clinical settings.
\end{abstract}

Keywords: Staphylococcus aureus, Methicillin Sensitive, Skin Swabs, School Going Children.

\section{Introduction}

Most children will have a skin infection at some time. Skin infections are a common reason for consultation in primary care and in dermatology practice (Harper $\mathbf{J}$ et al. 2000). Various studies done over the years suggest different types of organism involved. Most of the infections documented involve the superficial areas, rarely extending below the sub dermal region. Common skin infections include impetigo (Darmstadt et al. 1994), cellulitis, erysipelas (Currie BJ et al.2000), folliculitis, furuncles and carbuncles. The predominant strain causing infection varies by geographical location. In India multiple studies have been carried out to determine the etiology. Roughly $20 \%$ of outpatient dermatology visits are for bacterial skin infections in the western developed nations and the number is estimated higher in developing countries. Various parameters are involved in the outcome of the infection of which hygiene is also an important variable. It is indirectly correlated to the socio economic status (Currie BJ et al.2000). The aim of our study was to evaluate various types of bacteria causing skin infections in school going children of Udupi district in Karnataka State, India

\section{Materials and methods}

The study was a cross-sectional study. The study group consisted of school going children in Udupi district, Karnataka State, India. Consent was taken from the parents of respective children to screen for physical examination and to collect skin swabs.A total 236 children were screened for any skin lesions. A skin swab was collected from the site of lesions showing wound, cellulitis, nonhealing ulcer and other lesions evidencing possible active bacterial skin infection. The swabs were immediately immersed in Brain heart infusion broth (Hi-Media,Mumbai,India) and transported to the laboratory within one hour of collection(Mackie \& McCartney.1996) and incubated at $37^{\circ} \mathrm{C}$ for overnight or 48 hours (Which ever showed growth/ turbidity earlier).

The turbid BHI was sub-cultured onto a nutrient agar, blood agar and MacConkey agar, incubated aerobically for overnight. The colonies on these media were processed for the identification of the organisms according to (W.winn et al. 2006). Antibiotic sensitivity testing was done according to the guidelines recommended by Clinical and Laboratory Standards (CLSI) using Kirby-Bauer disc diffusion method. Staphylococcus aureus ATCC 29213 was 
used as control strain. Staphylococcus aureus was tested for methicillin using Oxacillin (S Ercis et al. 2008, M Gupta et al.2009, KB Anand et al.2009)

\section{Results}

A total of 53 children of $236(22.45 \%)$ were found to have abrasions or lesions from skin from which swabs were collected. The aerobic culture yielded pathogens in 8 subjects of $53(15.09 \%)$, of which 7 where Staphylococcus aureus $(13.2 \%)$ and one child had Klebsiella species (1.88\%).
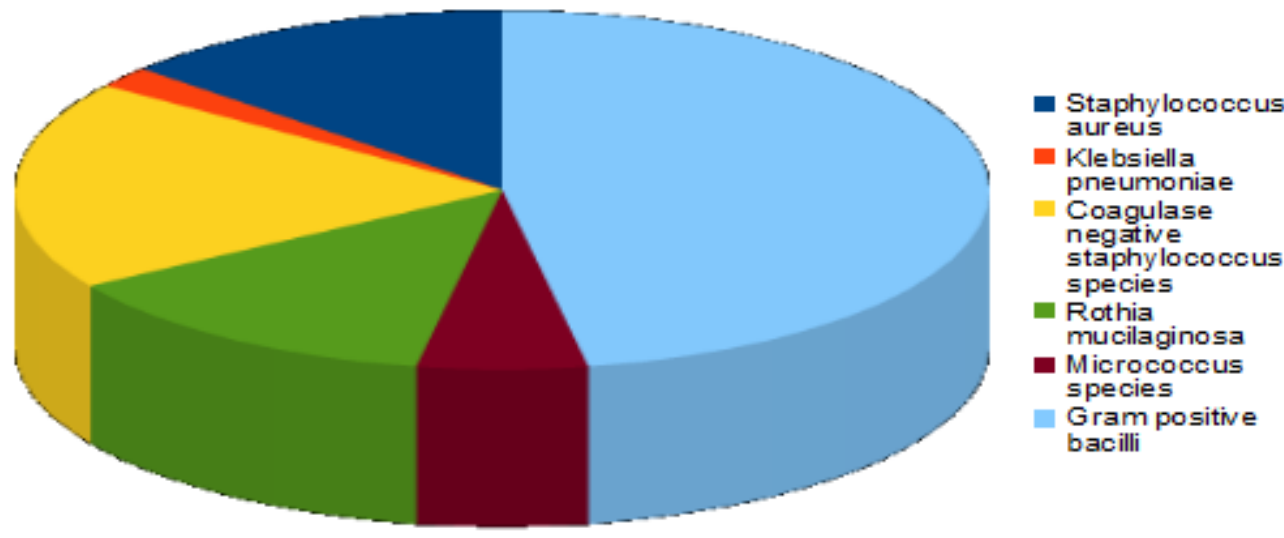

Fig. 1: Isolates from the Skin Swabs after Culture

Table1: Antibiotic Susceptibility Pattern of Staphylococcus Aureus

\begin{tabular}{|c|c|c|c|c|c|c|c|}
\hline \multicolumn{8}{|c|}{ S- Sensitive, R- Resistant } \\
\hline \multirow[b]{2}{*}{ Sample number } & \multirow[b]{2}{*}{ Site of lesion } & \multicolumn{5}{|c|}{ Sensitivity (Kirby- Bauer method disc diffusion method) } & \multirow[b]{2}{*}{ D-test } \\
\hline & & Oxacillin $(1 \mu \mathrm{g})$ & Cefazolin $(30 \mu \mathrm{g})$ & $\begin{array}{c}\text { Cefuroxime } \\
(30 \mu \mathrm{g})\end{array}$ & Erythromycin $(15 \mu \mathrm{g})$ & Clindamycin $(2 \mu \mathrm{g})$ & \\
\hline 7 & Leg & $\mathrm{S}$ & $\mathrm{S}$ & $\mathrm{R}$ & $\mathrm{R}$ & $\mathrm{S}$ & - \\
\hline 36 & Leg & $\mathrm{S}$ & $\mathrm{S}$ & $\mathrm{S}$ & $\mathrm{S}$ & $\mathrm{S}$ & - \\
\hline 37 & Leg & $\mathrm{S}$ & $\mathrm{S}$ & $\mathrm{S}$ & $\mathrm{S}$ & $\mathrm{S}$ & - \\
\hline 47 & Leg & $\mathrm{S}$ & $\mathrm{S}$ & $\mathrm{S}$ & $\mathrm{S}$ & $\mathrm{S}$ & - \\
\hline 75 & Hand & $\mathrm{S}$ & $\mathrm{S}$ & $\mathrm{S}$ & $\mathrm{R}$ & $\mathrm{S}$ & - \\
\hline 119 & Leg & $\mathrm{S}$ & $\mathrm{S}$ & $\mathrm{S}$ & $\mathrm{R}$ & $\mathrm{S}$ & - \\
\hline 143 & Fore-Head & $\mathrm{S}$ & $\mathrm{S}$ & $\mathrm{S}$ & $\mathrm{R}$ & $\mathrm{S}$ & + \\
\hline \multicolumn{2}{|c|}{$\%$ of sensitive organisms } & 100 & 100 & 85.71 & 42.85 & 100 & 85.71 \\
\hline
\end{tabular}

All the isolates were sensitive to Methicillin and Cefazolin. The Methicillin susceptibility was tested using $1 \mu \mathrm{g}$ Oxacillin discs. The clindamycin resistance though not detected by the disc diffusion test, D test was positive for one of the isolates.

The antibiotic susceptibility pattern of the Klebsiella pneumoniae

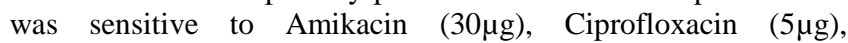
Ceftazidime $\quad(30 \mu \mathrm{g}), \quad$ Trimethoprim/sulphamethoxazole $(1.25 / 23.75 \mu \mathrm{g})$ and Gentamicin $(10 \mu \mathrm{g})$ and resistant to Ampicillin $(10 \mu \mathrm{g})$. They were also ESBL negative and sensitive to imipenem $(10 \mu \mathrm{g})$,

\section{Discussion}

Our study recovered $8(22.45 \%)$ organisms with established pathogenic role in skin infections of which, $7(87.5 \%)$ of the pathogenic isolates belonged to the Staphylococcus aureus. A meta-analysis done by (Cassandra D. Salgado et al in 2003) had shown that the pooled methicillin resistant Staphylococcus aureus (MRSA) colonization rate among community members was $1.3 \%$, but there was significant heterogeneity among study populations(Harrisons internal medicine.2008). In comparison, our study showed that the prevalence of MRSA was nil, though a negligible percentage may exist in community which may have been undetected. Our study was in good correlation with the study cited.

An important observation from our study was that the recovery of organisms from skin infections was related to personal hygiene practices, this was in concordance with the study conducted by (Currin BJ et al 2000). One of the subjects who showed multiple sites of infections, all the sites had positive culture for methicillin sensitive Staphylococcus aureus (MSSA) and the same susceptibility pattern and had history of poor personal hygienic practices. It was noted that all the isolates form this subject had a smaller zone (Same zone size from organisms isolated from different sites indicating same strain) of inhibition against the cefoxitin disc compared to other isolates from other subjects. This strain may progress to develop MRSA phenotype if treated with methicillin, though a genotype study was not done of this isolate and prediction is hence not possible.

Table1 reveals that in community acquired skin infections by Staphylococcus aureus the organisms were sensitive to methicillin and $1^{\text {st }}$ generation cephalosporin like cefazolin. There was increased resistance to the erythromycin. This was consistent with the data that had been established in many countries where erythromycin resistance was increasing in community acquired infections. We strongly have reasons to believe that the erythromycin resistance in community doesn't differ significantly from the hospital acquired infections with reference to staphylococcal infections. The data also shows that the $2^{\text {nd }}$ generation cephalosporins like Cefuroxime are less active against the Staphylococcus aureus, which was consistent with its established pharmacological properties (Chang CM et al.2008).

The Klebsiella pneumoniae was the only other pathogenic organism isolated $(14.28 \%)$. It is researched that the $K$ pneumoniae causes complicated skin and soft tissue infections (cSSTIs) in $<1 \%$ of cases (Principles and practice of clinical bacteriology.2006). It is hypothesized that few serotypes of $\mathrm{K}$ pneumoniae may occur as colonizer. Though serotyping was not performed, the candidate from whom the isolate was obtained had no other clinical manifestations. Generally, pathogenic Klebsiella causing infection of skin, progresses to form a more invasive lesion such as infection of extremities or soft tissue. In our case this was not evident. Hence we reason that the isolate probably belonged to a nonpathogenic serotype.

Recovery of other organisms which constituted normal flora (chart 1), from the skin cultures provided an indication of good personal 
hygiene. The infection was often due to skin abrasions, which were often seen in children of this age group due to outdoor physical activity. If the hygiene was good, the skin would be devoid of pathogenic organisms and wound would be contaminated with skin flora which spontaneously would have resolved. Failure to isolate pathogenic organisms from 45 subjects $(84.9 \%)$ indicated good overall personal hygiene from the children.

\section{Conclusion}

Though Staphylococcus aureus was the common organism isolated, all were sensitive to methicillin but high prevalence of erythromycin resistance of the Staphylococcus aureus in the community demands proper screening of isolates for resistance in clinical settings. A future study on the same population which includes subjects from wider geographic area should be considered.

\section{References}

[1] Harper J, Oranje A, Prose N, eds. Textbook of pediatric dermatology. Oxford: Blackwell, 2000-Prime chapters on cutaneous infections of childhood.

[2] Darmstadt G, Lane A. Impetigo an overview. Pediatric Dermatology. 1994; 11:293-303 http://dx.doi.org/10.1111/j.1525 1470.1994.tb00092.x.

[3] Currie BJ, Carapetis JR. Skin infections and infestations in Aboriginal communities in northern Australia. Australas J Dermatol 2000; 41:139-43. http://dx.doi.org/10.1046/j.1440-0960.2000.00417.x.

[4] Mackie and Mc Cartney, Practical medical microbiology, 1996, 14th ed, Churchill Livingstone.

[5] Winn W, Jr Allen S, Janda W et al: Koneman's color atlas and textbook of diagnostic microbiology 6th edition.2006 Philadelphia USA.

[6] Performance standards for antimicrobial susceptibility testing:20th informational supplement,Clinical and laboratory standards Institute (CLSI).M100-S20;Vol 30, No1,Wayne PA;Clinical and laboratory standards institute 2010. Wayne, PA.

[7] S Rallapalli, S Verghese, RS Verma; Validation of multiplex PCR strategy for simultaneous detection and identification of methicillin resistant Staphylococcus aureus, IJMM, 2008,vol 26, 361-364.

[8] M Gupta, NP Singh, A Kumar, IR Kaur; Cefoxitin disk diffusion test Better predictor of methicillin resistance in Staphylococcus aureus 2009Vol: 27: 379-380.

[9] S Ercis, B Sancak, G Hascelik; A comparison of PCR detection of $M e c a$ with oxacillin disk susceptibility testing in different media and sceptor automated system for both Staphylococcus aureus and coagulase-negative staphylococci isolates,IIJM, 2008, Vol2, 21-24.

[10]KB Anand, P Agrawal, S Kumar, K Kapila, Comparison of cefoxitin disc diffusion test, oxacillin screen agar, and PCR for mecA gene for detection of MRSA, IIJM, 2009, Vol27, 27-29.

[11]Cassandra D. Salgado, Barry M. Farr and David P. Calfee; Community-Acquired MRSA: A Meta-Analysis of Prevalence and Risk Factors, Clinical Infectious Diseases 2003; 36:131-139. http://dx.doi.org/10.1086/345436.

[12]Harrisons internal medicine, Fauci etal, 2008, 17th edition, Mc Graw Hill publications.

[13]Chang CM, Lee HC, Lee NY, Lee IW, Wu CJ, Chen PL, Lee CC, Ko NY, Ko WC; Community-acquired Klebsiella pneumoniae complicated skin and soft-tissue infections of extremities: Emphasis on cirrhotic patients and gas formation. Infection, 2008 Aug; 36(4):328-34 http://dx.doi.org/10.1007/s15010-008-7272-3

[14]Principles and practice of Clinical Bacteriology, 2006, 2nd edition Stephen H. Gillespie. 